\title{
RESPONSE OF CAMBIAL AND SHOOT GROWTH IN TREES TREATED WITH PACLOBUTRAZOL by Shuju Bai ${ }^{1}$, William Chaney ${ }^{2}$, and Yadong $Q^{i}$
}

\begin{abstract}
Three experiments were conducted to investigate the effect of paclobutrazol on shoot and cambial growth of nine tree species located in Indiana and Louisiana, U.S. In Experiment I, white oak (Quercus alba L.) and red oak (Q. rubra L.) in Indiana were treated in April 1995 with paclobutrazol using the soil drench method. In Experiment II, paclobutrazol was applied in April 1996, using the soil injection method, to eight species growing in Indiana: white oak, red oak, sweetgum (Liquidambar styraciflua L.), black walnut (Juglans nigra L.), European black alder (Alnus glutinosa L.), yellow poplar (Liriodendron tulipifera L.), white ash (Fraxinus americana L.), and white pine (Pinus strobus L.). In Experiment III, two species, sweetgum and cherrybark oak (Q. falcata var. pagodaefolia L.) in Louisiana, were treated with paclobutrazol by the soil drench method in March 1997. At the end of the 1998 growing season, annual shoot growth and annual xylem ring increment were measured for each of the two to four growing seasons after treatment in the three experiments. The effects of paclobutrazol on cambial growth and annual shoot growth at various positions along the trunk and in the canopy varied with species and treatment duration. Paclobutrazol reduced cambial growth in white oak, red oak, cherrybark oak, sweetgum, European black alder, and white pine with the amount varying among species, vertical location in the tree, and year after treatment.
\end{abstract}

Key Words. Paclobutrazol (PBZ); growth retardant; cambial growth; annual shoot growth; xylem ring increment.

Paclobutrazol (PBZ), which inhibits gibberellin synthesis and consequently cell elongation (Kimball 1990; Grossmann 1992; Rademacher 2000), is known to reduce the growth of woody plants and has been used successfully for this purpose by horticulturists and utility arborists (Keever et al. 1990; Werblow 1998; Fletcher et al. 2000). The use of PBZ by utility arborists lengthens the time between trimming cycles, reduces the amount of time at the job site, and lowers the amount of biomass removed during trimming for many tree species (Redding et al. 1994; Burch et al. 1996). Shoot growth reduction along with other benefits to tree health reported in recent years has led to the expansion of PBZ use solely by utility foresters to its use by commercial arborists for management of other trees in the urban landscape (Chaney 2003).

Although PBZ is known to suppress shoot growth of most tree species (Davis and Curry 1991) and to increase tolerance to drought conditions and resistance to fungal organisms (Chaney et al. 1996; Fletcher et al. 2000), the response of cambial growth in PBZ-treated trees has not been clearly elucidated. Because the principal focus of research with growth retardants such as PBZ has been on growth in height, only a few observations have been recorded concerning growth in diameter. Previous research, although limited in scope, has generally indicated a suppression of cambial growth (Gilliam et al. 1988; Estabrooks 1993; Schnurr et al. 1996). Growth in diameter of trees is a major cause of damage to hardscape in urban areas, accounting for a significant portion of annual tree program expenditures by municipalities (McPherson and Peper 1995). Hence, the main objective of this study was to determine the effect of the tree growth regulator PBZ on cambial growth of several tree species growing in the different environmental conditions of Indiana and Louisiana, U.S. Growth in length of terminal and lateral shoots also was measured to relate the acknowledged reduction of primary growth to any effect on secondary or cambial growth.

\section{MATERIALS AND METHODS}

Trees used in this study were in two locations; one at the Horticulture Farm at Southern University and A\&M College in Baton Rouge, Louisiana, U.S., and the other at Martell Experimental Forest near Purdue University in West Lafayette, Indiana, U.S. . Three experiments were designed, two at the Indiana and one at the Louisiana site, using a combination of nine tree species. For Experiment I conducted in Indiana, two species, red oak (Quercus rubra L.) and white oak ( 2 . alba L. [10 cm (4 in.) average caliper] were treated by the soil drench method in April 1995. Experiment II, also in Indiana, involved eight species ranging from 4 to $8 \mathrm{~cm}$ [1.6 to $3.2 \mathrm{in}$.] caliper treated in April 1996 using the soil injection method: black walnut (Juglans nigra L.), European black alder (Alnus glutinosa L.), red oak, sweetgum (Liquidambar styraciflua L.), white ash (Fraxinus americana L.), white oak, white pine (Pinus strobus L.), and yellow poplar (Liriodendron tulipifera L.). Experiment III, conducted in Louisiana, involved two species treated in March 1997 using the soil drench method: cherrybark oak (Q. falcata var. pagodaefolia Elliott) and sweetgum [4 cm (1.6 in.) average caliper]. 
Twenty (Experiment I), 12 (Experiment II), and 14 (Experiment III) plantation trees of each species growing at $2 \times 2 \mathrm{~m}[6.6 \times 6.6 \mathrm{ft}]$ spacing were selected based on their size and condition in 1995, 1996, and 1997, respectively. Adjacent trees were not selected for the studies to avoid root interaction and the unintended exposure to PBZ treatment. Half of the trees for each species used in the three experiments was randomly assigned to each of two different treatment groups. The treatments were basal drench or soil injection with PBZ formulated as Profile 2SC or water as a control in complete random experimental designs. The dose of PBZ applied was $9.6 \mathrm{~g}$ active ingredient (g a.i.) (0.34 oz) per tree in all the experiments except the sweetgum trees in Louisiana, which were treated with $4.8 \mathrm{~g}$ a.i. ( $0.17 \mathrm{oz}$.) per tree.

For Experiment I, the annual shoot growth for the 1995, 1996, 1997, and 1998 growing seasons was measured on the main stem, on four lateral shoots in the upper crown, and on four lateral shoots in the lower crown of each tree. For Experiment II, annual shoot growth for the 1996, 1997, and 1998 growing seasons was measured on the main stem and four lateral shoots of each tree. For Experiment III, the annual shoot growth for the 1997 and 1998 growing seasons was measured as in Experiment II. The amount of annual growth was apparent by locating the terminal bud scale scars on branches. All of the species used normally produce only one flush of growth each growing season. Shoot growth for European black alder is not reported because it was impossible to locate with certainty the bud scale scars on this species.

Cross-sections of the trunk were removed approximately $50 \mathrm{~cm}$ (20 in.) from the base of each tree for observations of annual xylem ring width in Experiment I. In Experiment II, three cross-sections were removed from each selected tree. One was approximately $10 \mathrm{~cm}$ ( 4 in.) from the base; the second was either 2 or $4 \mathrm{~m}$ (6.6 or $13.2 \mathrm{ft}$ ) from the base (determined by the height of the tree), and the third was from wherever the cross-section would include the annual rings of xylem for the 1995 through 1998 growing seasons. In Experiment III, an increment borer was used to obtain a wood sample for determining the ring width at a height of $10 \mathrm{~cm}$ (4 in.) from the base of each tree. An increment core also was taken from the highest point in the trees that would include the annual rings of xylem for the 1996 through 1998 growing seasons. Increment core samples were taken from only two heights in the trees in Louisiana because the trees were not tall enough to allow for additional sampling.
Each cross-section was sanded smooth with a handheld orbital sander, and each increment core was cut with a sharp blade to make a flat surface to facilitate microscopic viewing of the annual rings of xylem. Xylem ring widths for the year before PBZ treatment through the 1998 growing season were measured along four radii at approximately right angles for each cross-section using an Acu-rite III (Jamestown, NY) digital measuring system. Wood sections were placed on the table of the manually mobilized scale assembly and viewed through a lens with $10 \times$ magnification. The console was set for incremental measurement mode, and it gave a digital readout of the distance across each xylem ring with an accuracy of $0.001 \mathrm{~mm}$ (0.00004 in.).

Data were analyzed using analysis of variance and differences between means were determined using Tukey's Studentized Range (HSD) test, $P \leq 0.05$.

\section{RESULTS \\ Annual Shoot Growth}

Annual growth was not affected the first growing season after PBZ treatment in either red or white oak grown in Indiana (Experiment I), but it was markedly reduced in the central leader and in shoots of the upper and lower crown of white oak for the next three growing seasons (Table 1). Significant
Table 1. Annual and total shoot growth $(\mathrm{cm})$ for 4 years and total reduction expressed as a percentage of untreated controls for white and red oak in Indiana untreated and treated in April 1995 with paclobutrazol using the soil drench method.

\begin{tabular}{lllllll}
\hline & \multicolumn{4}{c}{ Year } & $\begin{array}{l}\text { 4-year } \\
\text { total }\end{array}$ & $\begin{array}{l}\text { Percentage } \\
\text { reduction }\end{array}$ \\
\cline { 2 - 6 } Treatment & 1995 & 1996 & 1997 & 1998 &
\end{tabular}

\section{White oak}

Central leader

$\begin{array}{llllll}\text { Control } & 42.9 \mathrm{a} & 46.6 \mathrm{a} & 41.1 \mathrm{a} & 40.9 \mathrm{a} & 170.5 \mathrm{a}\end{array}$

$\begin{array}{llllll}\text { Treated } & 39.0 \mathrm{a} & 20.0 \mathrm{~b} & 6.6 \mathrm{~b} & 10.5 \mathrm{~b} & 76.1 \mathrm{~b}\end{array}$

55

Shoots in upper crown

Control $\quad 38.5$ a $\quad 36.9$ a $\quad 39.6$ a $\quad 31.3$ a $\quad 146.3 a$

Shoots in lower crown

Control $\quad 28.2 \mathrm{a} \quad 34.1 \mathrm{a} \quad 32.8 \mathrm{a} \quad 26.6 \mathrm{a} \quad 121.7 \mathrm{a}$

$\begin{array}{llllll}\text { Treated } & 20.2 \mathrm{a} & 9.3 \mathrm{~b} & 3.4 \mathrm{~b} & 5.8 \mathrm{~b} & 38.7 \mathrm{~b}\end{array}$

68

Red oak

Central leader

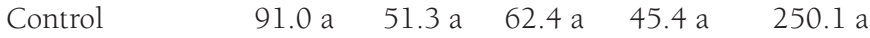

Treated $\quad 76.9 \mathrm{a} \quad 26.3 \mathrm{~b} \quad 12.5 \mathrm{~b} \quad 14.9 \mathrm{~b} \quad 130.6 \mathrm{~b}$

48

Shoots in upper crown

$\begin{array}{llllll}\text { Control } & 72.5 \mathrm{a} & 42.3 \mathrm{a} & 40.6 \mathrm{a} & 37.4 \mathrm{a} & 192.8 \mathrm{a}\end{array}$

$\begin{array}{lllllll}\text { Treated } & 67.4 \mathrm{a} & 18.6 \mathrm{~b} & 8.9 \mathrm{~b} & 12.3 \mathrm{~b} & 107.2 \mathrm{~b} & 44\end{array}$

Shoots in lower crown

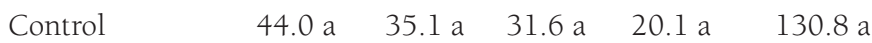

$\begin{array}{lllllll}\text { Treated } & 51.5 \mathrm{a} & 20.0 \mathrm{a} & 5.2 \mathrm{~b} & 7.2 \mathrm{~b} & 83.9 \mathrm{~b} & 36\end{array}$

Pairs of values followed by the same lowercase letter are not significantly different at the $P \leq 0.05$ level. 
reduction in annual shoot growth also was observed in the central leader and the upper crown of red oak for the second and the next two growing seasons. However, in the lower crown of red oak, significant reduction in annual shoot growth occurred only in the third and fourth years after PBZ treatment. Nevertheless, the total growth in shoot length was reduced throughout the crown in both species by as much as $36 \%$ to $68 \%$ compared to the untreated controls (Table 1 ).

Annual shoot growth of the central leader of the seven species investigated in Experiment II in Indiana showed large variations among species and years in response to soil injection of PBZ (Table 2). Annual growth of the central leader of sweetgum and white pine was reduced for the three growing seasons after PBZ treatment. Reduction of annual growth of the central leader of red oak, white ash, and white oak occurred in the first and second growing seasons after treatment, but in the third season, growth suppression disappeared (Table 2). In yellow poplar, inhibition occurred only in the first season after treatment. No inhibition of shoot growth was observed through the three growing seasons in black walnut. Total growth of the central leader over the 3year study period was significantly suppressed in only sweetgum (99\%), white oak (46\%), and white pine (30\%) (Table 2).

For growth in length of the lateral shoots in the seven species in Experiment II in Indiana, only PBZ-treated sweetgum and white oak trees were suppressed for the three growing seasons after treatment, resulting in a total reduction in three growing seasons of $99 \%$ and $66 \%$, respectively. Shoot growth of lateral branches of white pine was inhibited only in the second and third growing seasons, but this growth suppression was adequate to result in 19\% less growth of PBZ-treated trees during the 3-year study period (Table 2). Growth reduction in red oak and yellow poplar occurred only the first growing season after treatment. No growth suppression was found in black walnut (Table 2).

Table 2. Annual and total shoot growth $(\mathrm{cm})$ for 3 years and total reduction expressed as a percentage of untreated controls of seven species of trees in Indiana untreated and treated in April 1996 with paclobutrazol using the soil injection method.

\begin{tabular}{|c|c|c|c|c|c|c|c|c|c|c|c|}
\hline \multirow{2}{*}{ Treatment } & \multicolumn{3}{|c|}{ Year } & \multirow{2}{*}{$\begin{array}{l}\text { 3-year } \\
\text { total }\end{array}$} & \multirow{2}{*}{$\begin{array}{l}\text { Percentage } \\
\text { reduction }\end{array}$} & \multirow[b]{2}{*}{ Treatment } & \multicolumn{3}{|c|}{ Year } & \multirow{2}{*}{$\begin{array}{l}\text { 3-year } \\
\text { total }\end{array}$} & \multirow{2}{*}{$\begin{array}{l}\text { Percentage } \\
\text { reduction }\end{array}$} \\
\hline & 1996 & 1997 & 1998 & & & & 1996 & 1997 & 1998 & & \\
\hline \multicolumn{6}{|l|}{ Black walnut } & \multicolumn{6}{|l|}{ White oak } \\
\hline \multicolumn{6}{|l|}{ Central leader } & \multicolumn{6}{|l|}{ Central leader } \\
\hline Control & $27.5 \mathrm{a}$ & $13.5 \mathrm{a}$ & $7.5 \mathrm{a}$ & $48.5 \mathrm{a}$ & & Control & $37.3 \mathrm{a}$ & $32.0 \mathrm{a}$ & $28.7 \mathrm{a}$ & $98.0 \mathrm{a}$ & \\
\hline Treated & $12.5 \mathrm{a}$ & 8.9 a & $6.8 \mathrm{a}$ & $28.2 \mathrm{a}$ & 42 & Treated & $18.2 \mathrm{~b}$ & $13.7 \mathrm{~b}$ & $20.7 \mathrm{a}$ & $52.6 \mathrm{~b}$ & 46 \\
\hline \multicolumn{6}{|l|}{ Lateral shoots } & \multicolumn{6}{|l|}{ Lateral shoots } \\
\hline Control & $10.0 \mathrm{a}$ & $10.5 \mathrm{a}$ & $8.2 \mathrm{a}$ & $28.7 \mathrm{a}$ & & Control & $41.7 \mathrm{a}$ & $28.7 \mathrm{a}$ & $32.8 \mathrm{a}$ & $103.2 \mathrm{a}$ & \\
\hline Treated & $7.0 \mathrm{a}$ & $5.6 \mathrm{a}$ & $4.8 \mathrm{a}$ & $17.4 \mathrm{a}$ & 39 & Treated & $14.7 \mathrm{~b}$ & $10.0 \mathrm{~b}$ & $10.2 \mathrm{~b}$ & $34.9 \mathrm{~b}$ & 66 \\
\hline \multirow{2}{*}{\multicolumn{6}{|c|}{$\begin{array}{l}\text { Red oak } \\
\text { Central leader }\end{array}$}} & \multirow{2}{*}{\multicolumn{6}{|c|}{$\begin{array}{l}\text { White pine } \\
\text { Central leader }\end{array}$}} \\
\hline & & & & & & & & & & & \\
\hline Control & $71.7 \mathrm{a}$ & $45.7 \mathrm{a}$ & $31.2 \mathrm{a}$ & $148.6 \mathrm{a}$ & & Control & $95.3 \mathrm{a}$ & $82.3 \mathrm{a}$ & $89.8 \mathrm{a}$ & $267.4 \mathrm{a}$ & \\
\hline Treated & $64.3 \mathrm{~b}$ & $31.3 \mathrm{~b}$ & $28.1 \mathrm{a}$ & $123.7 \mathrm{a}$ & 17 & Treated & $84.0 \mathrm{~b}$ & $48.0 \mathrm{~b}$ & $55.7 \mathrm{~b}$ & $187.7 \mathrm{~b}$ & 30 \\
\hline \multicolumn{6}{|c|}{ Lateral shoots } & \multicolumn{6}{|c|}{ Lateral shoots } \\
\hline Control & $55.4 \mathrm{a}$ & $23.4 \mathrm{a}$ & $21.5 \mathrm{a}$ & $100.3 \mathrm{a}$ & & Control & $55.4 \mathrm{a}$ & $46.5 \mathrm{a}$ & $37.2 \mathrm{a}$ & $139.1 \mathrm{a}$ & \\
\hline Treated & $40.1 \mathrm{~b}$ & $17.5 \mathrm{a}$ & $16.2 \mathrm{a}$ & $73.8 \mathrm{a}$ & 26 & Treated & $50.0 \mathrm{a}$ & $34.7 \mathrm{~b}$ & $28.6 \mathrm{~b}$ & $113.3 \mathrm{~b}$ & 19 \\
\hline \multirow{2}{*}{\multicolumn{6}{|c|}{$\begin{array}{l}\text { Sweetgum } \\
\text { Central leader }\end{array}$}} & \multicolumn{6}{|c|}{ Yellow poplar } \\
\hline & & & & & & Central leader & & & & & \\
\hline Control & $51.5 \mathrm{a}$ & $54.7 \mathrm{a}$ & $67.0 \mathrm{a}$ & $173.2 \mathrm{a}$ & & Control & $53.7 \mathrm{a}$ & $43.0 \mathrm{a}$ & $63.3 \mathrm{a}$ & $160.0 \mathrm{a}$ & \\
\hline Treated & $0.8 \mathrm{~b}$ & $0.3 \mathrm{~b}$ & $0.3 \mathrm{~b}$ & $1.4 \mathrm{~b}$ & 99 & Treated & $37.0 \mathrm{~b}$ & $44.1 \mathrm{a}$ & $59.5 \mathrm{a}$ & $140.6 \mathrm{a}$ & 12 \\
\hline \multicolumn{6}{|l|}{ Lateral shoots } & \multicolumn{6}{|l|}{ Lateral shoots } \\
\hline Control & $34.0 \mathrm{a}$ & $27.7 \mathrm{a}$ & $21.7 \mathrm{a}$ & $83.4 \mathrm{a}$ & & Control & $32.3 \mathrm{a}$ & $28.3 \mathrm{a}$ & $24.7 \mathrm{a}$ & $85.3 \mathrm{a}$ & \\
\hline Treated & $0.7 \mathrm{~b}$ & $0.3 \mathrm{~b}$ & $0.2 \mathrm{~b}$ & $1.2 \mathrm{~b}$ & 99 & Treated & $21.8 \mathrm{~b}$ & $22.4 \mathrm{a}$ & $18.6 \mathrm{a}$ & $62.8 \mathrm{a}$ & 26 \\
\hline \multirow{2}{*}{\multicolumn{12}{|c|}{$\begin{array}{l}\text { White ash } \\
\text { Central leader }\end{array}$}} \\
\hline & & & & & & & & & & & \\
\hline Control & $44.8 \mathrm{a}$ & $37.5 \mathrm{a}$ & $37.5 \mathrm{a}$ & $119.8 \mathrm{a}$ & & & & & & & \\
\hline Treated & $31.3 \mathrm{~b}$ & $26.3 \mathrm{~b}$ & $35.0 \mathrm{a}$ & $92.6 \mathrm{a}$ & 23 & & & & & & \\
\hline \multicolumn{12}{|l|}{ Lateral shoots } \\
\hline Control & $31.0 \mathrm{a}$ & $20.5 \mathrm{a}$ & $15.2 \mathrm{a}$ & $66.7 \mathrm{a}$ & & & & & & & \\
\hline Treated & $24.0 \mathrm{a}$ & $17.5 \mathrm{a}$ & $13.9 \mathrm{a}$ & $55.4 \mathrm{a}$ & 17 & & & & & & \\
\hline
\end{tabular}

Pairs of values followed by the same lowercase letter are not significantly different at the $P \leq 0.05$ level. 
Table 3. Annual and total shoot growth (cm) for 2 years and total reduction expressed as a percentage of untreated controls of sweetgum and cherrybark oak in Louisiana untreated and treated in March 1997 with paclobutrazol using the soil drench method.

\begin{tabular}{lrrrr}
\hline & \multicolumn{2}{c}{ Year } & $\begin{array}{l}\text { 2-year } \\
\text { total }\end{array}$ & $\begin{array}{l}\text { Percentage } \\
\text { reduction }\end{array}$ \\
\cline { 2 - 4 } Treatment & 1997 & 1998 & & \\
Sweetgum & & & & \\
Central leader & & & & \\
Control & $83.5 \mathrm{a}$ & $89.3 \mathrm{a}$ & $172.8 \mathrm{a}$ & \\
Treated & $6.5 \mathrm{~b}$ & $1.4 \mathrm{~b}$ & $7.9 \mathrm{~b}$ & 95 \\
Lateral shoots & & & & \\
Control & $33.3 \mathrm{a}$ & $28.0 \mathrm{a}$ & $61.3 \mathrm{a}$ & \\
Treated & $1.4 \mathrm{~b}$ & $1.1 \mathrm{~b}$ & $2.5 \mathrm{~b}$ & 96 \\
Cherrybark oak & & & & \\
Central leader & & & & \\
Control & $36.1 \mathrm{a}$ & $30.0 \mathrm{a}$ & $66.1 \mathrm{a}$ & \\
Treated & $31.2 \mathrm{a}$ & $4.2 \mathrm{~b}$ & $35.4 \mathrm{~b}$ & 46 \\
Lateral shoots & & & & \\
Control & $24.8 \mathrm{a}$ & $25.0 \mathrm{a}$ & $49.8 \mathrm{a}$ & \\
Treated & $23.9 \mathrm{a}$ & $4.8 \mathrm{~b}$ & $28.7 \mathrm{~b}$ & 42 \\
\hline Pats of vacs & & & & \\
\hline
\end{tabular}

Pairs of values followed by the same lowercase letter are not significantly different at the $P \leq 0.05$ level.

Table 4. Annual xylem ring increment $(\mathrm{mm}) 50 \mathrm{~cm}$ from the ground line for white and red oak for the year prior to treatment and for 4 years after treatment with paclobutrazol in April 1995 using the soil drench method, with total reduction expressed as a percentage of untreated controls.

\begin{tabular}{|c|c|c|c|c|c|c|c|}
\hline \multirow[b]{3}{*}{ Treatment } & \multicolumn{5}{|c|}{ Year } & \multirow{3}{*}{$\begin{array}{l}\text { Total } \\
1995-98\end{array}$} & \multirow{3}{*}{$\begin{array}{l}\text { Percentage } \\
\text { reduction }\end{array}$} \\
\hline & Prior & & ffter tr & atment & & & \\
\hline & 1994 & 1995 & 1996 & 1997 & 1998 & & \\
\hline
\end{tabular}

\section{White oak}

$\begin{array}{lllllll}\text { Control } & 4.95 \mathrm{a} & 4.43 \mathrm{a} & 5.06 \mathrm{a} & 4.50 \mathrm{a} & 6.11 \mathrm{a} & 20.10 \mathrm{a}\end{array}$

Treated $\quad 5.30 \mathrm{a} \quad 1.60 \mathrm{~b} \quad 0.99 \mathrm{~b} \quad 0.70 \mathrm{~b} \quad 0.71 \mathrm{~b} \quad 4.00 \mathrm{~b}$

Red oak

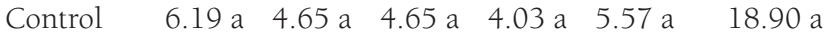

Treated $\quad 6.24 \mathrm{a} \quad 2.68 \mathrm{~b} \quad 1.78 \mathrm{~b} \quad 1.37 \mathrm{~b} \quad 1.81 \mathrm{~b} \quad 7.64 \mathrm{~b}$

Pairs of values followed by the same lowercase letter are not significantly different at the $P \leq 0.05$ level.

In Louisiana, annual shoot growth of sweetgum was markedly inhibited in both the central leader and the lateral shoots for the two growing seasons after PBZ treatment, resulting in total growth suppression of $95 \%$ and $96 \%$, respectively (Table 3). For cherrybark oak, inhibition occurred only in the second growing season after treatment, and the total reduction in growth in 2 years was significant, but less than in sweetgum.

\section{Annual Xylem Ring Increment}

In Experiment I, the width of the annual ring of xylem produced $50 \mathrm{~cm}$ (20 in.) up from the base of trees was significantly reduced in both white and red oak during the first growing season after PBZ treatment by the soil drench method. This suppression of cambial activity in treated trees persisted for the four growing seasons of the study, resulting in a total reduction of cambial growth in white and red oak of $80 \%$ and $60 \%$, respectively (Table 4 ).

Xylem accumulation at the base of the trunk of trees treated in spring 1996 using the soil injection method (Experiment II) showed that PBZ suppressed annual ring increment in sweetgum for the first and second growing seasons after treatment, and in white oak for the second and third seasons (Table 5). Reduction of xylem accumulation occurred in white pine in only the first growing season after treatment and in European black alder in the third growing season after treatment. No growth suppression near the base of the trunk was observed in the other species (Table 5).

A similar pattern of xylem growth reduction in sweetgum and white oak also occurred in the cross-sections 2 or $4 \mathrm{~m}(6.6$ or $13.2 \mathrm{ft})$ above the ground line (Table 5). Treated European black alder had significantly reduced annual ring growth for the three growing seasons after PBZ treatment. In addition, PBZ suppressed annual ring increment of white pine in the second growing season. No growth reduction 2 or $4 \mathrm{~m}$ high was found in the other species investigated (Table 5).

Annual ring increment in the upper crown in the cross sections of the trunk that included four years of xylem growth of the eight species treated in 1996 using the soil injection method (Experiment II) also are shown in Table 5. PBZ-treated sweetgum trees had less xylem increment than control trees for the three growing seasons after treatment. Growth suppression also occurred in white oak and white pine during the second and the third growing seasons. Annual ring increment in the upper crown in the other species was not affected by PBZ (Table 5).

Total cambial growth for the three years following PBZ treatment was significantly reduced $(P \leq 0.05)$ only for sweetgum and white oak. There also was a 3 -year reduction in the mid-trunk section of European black alder and in the cross-section within the crown of white pine (Table 5).

In Louisiana (Experiment III), the annual ring increment of cherrybark oak was reduced by PBZ at both the base and within the crown for the two growing seasons after PBZ 
Table 5. Annual xylem ring increment $(\mathrm{mm})$ for the year prior to treatment and for 3 years after treatment with paclobutrazol in April 1996 using the soil injection method on eight tree species growing in Indiana, with total reduction expressed as a percentage of untreated controls.

\begin{tabular}{|c|c|c|c|c|c|c|c|c|c|c|c|c|c|}
\hline \multirow[b]{3}{*}{ Treatment } & \multicolumn{4}{|c|}{ Year } & \multirow{3}{*}{$\begin{array}{l}\text { Total } \\
\text { 1996-98 }\end{array}$} & \multirow{3}{*}{$\begin{array}{l}\text { Percentage } \\
\text { reduction }\end{array}$} & \multirow[b]{3}{*}{ Treatment } & \multicolumn{4}{|c|}{ Year } & \multirow{3}{*}{$\begin{array}{l}\text { Total } \\
1996-98\end{array}$} & \multirow{3}{*}{$\begin{array}{l}\text { Percentage } \\
\text { reduction }\end{array}$} \\
\hline & \multirow{2}{*}{$\frac{\text { Prior }}{1995}$} & \multicolumn{3}{|c|}{ After treatment } & & & & Prior & $\mathrm{Aft}$ & r treatme & & & \\
\hline & & 1996 & 1997 & 1998 & & & & $\overline{1995}$ & 1996 & 1997 & 1998 & & \\
\hline \multicolumn{7}{|c|}{ Black walnut } & \multicolumn{7}{|l|}{ White ash } \\
\hline \multicolumn{7}{|c|}{ Within crown } & \multicolumn{7}{|c|}{ Within crown } \\
\hline Control & $1.36 \mathrm{a}$ & $1.38 \mathrm{a}$ & $1.06 \mathrm{a}$ & $1.08 \mathrm{a}$ & $3.52 \mathrm{a}$ & & Control & $1.55 \mathrm{a}$ & $1.63 \mathrm{a}$ & $1.95 \mathrm{a}$ & $2.07 \mathrm{a}$ & $5.65 \mathrm{a}$ & \\
\hline Treated & $0.82 \mathrm{a}$ & $1.28 \mathrm{a}$ & $0.99 a$ & $0.94 \mathrm{a}$ & $3.21 \mathrm{a}$ & 9 & Treated & $1.09 \mathrm{a}$ & $1.27 \mathrm{a}$ & $1.32 \mathrm{a}$ & $1.48 \mathrm{a}$ & $4.07 \mathrm{a}$ & 28 \\
\hline \multicolumn{7}{|c|}{ Mid-trunk (2-4 m high) } & \multicolumn{7}{|c|}{ Mid-trunk (2-4 m high) } \\
\hline Control & $2.05 \mathrm{a}$ & $2.44 \mathrm{a}$ & $1.46 \mathrm{a}$ & $1.67 \mathrm{a}$ & $5.57 \mathrm{a}$ & & Control & $2.21 \mathrm{a}$ & $2.11 \mathrm{a}$ & $2.21 \mathrm{a}$ & $2.15 \mathrm{a}$ & $6.47 \mathrm{a}$ & \\
\hline Treated & $1.74 \mathrm{a}$ & $1.54 \mathrm{a}$ & $1.14 \mathrm{a}$ & $1.40 \mathrm{a}$ & $4.08 \mathrm{a}$ & 27 & Treated & $1.39 \mathrm{a}$ & $1.62 \mathrm{a}$ & $1.55 \mathrm{a}$ & $1.66 \mathrm{a}$ & $4.83 \mathrm{a}$ & 25 \\
\hline \multicolumn{7}{|c|}{ Base of trunk (10 cm above ground line) } & \multicolumn{7}{|c|}{ Base of trunk (10 cm above ground line) } \\
\hline Control & $1.86 \mathrm{a}$ & $1.42^{\circ} \mathrm{a}$ & $1.25 \mathrm{a}$ & $1.66 \mathrm{a}$ & $4.33 \mathrm{a}$ & & Control & $2.03 \mathrm{a}$ & $2.25 \mathrm{a}$ & $1.92 \mathrm{a}$ & $2.25 \mathrm{a}$ & $6.42 \mathrm{a}$ & \\
\hline Treated & $2.06 \mathrm{a}$ & $1.61 \mathrm{a}$ & $1.05 \mathrm{a}$ & $1.04 \mathrm{a}$ & $3.70 \mathrm{a}$ & 15 & Treated & $1.67 \mathrm{a}$ & $1.57 \mathrm{a}$ & $1.30 \mathrm{a}$ & $1.71 \mathrm{a}$ & $4.58 \mathrm{a}$ & 29 \\
\hline \multicolumn{7}{|c|}{ European black alder } & \multicolumn{7}{|l|}{ White oak } \\
\hline \multicolumn{7}{|c|}{ Within crown } & Within cron & & & & & & \\
\hline Control & $2.06 \mathrm{a}$ & $1.84 \mathrm{a}$ & $1.46 \mathrm{a}$ & $1.50 \mathrm{a}$ & $4.80 \mathrm{a}$ & & Control & $2.42 \mathrm{a}$ & $2.17 \mathrm{a}$ & $1.84 \mathrm{a}$ & $2.68 \mathrm{a}$ & $6.69 \mathrm{a}$ & \\
\hline Treated & $2.59 \mathrm{a}$ & $1.20 \mathrm{a}$ & $1.11 \mathrm{a}$ & $1.29 \mathrm{a}$ & $3.60 \mathrm{a}$ & 25 & Treated & $2.46 \mathrm{a}$ & $1.08 \mathrm{a}$ & $0.80 \mathrm{~b}$ & $0.97 \mathrm{~b}$ & $2.85 \mathrm{~b}$ & 57 \\
\hline Mid-trunk & (2-4 $m h$ & & & & & & Mid-trunk & $2-4 m h$ & & & & & \\
\hline Control & $5.80 \mathrm{a}$ & $5.00 \mathrm{a}$ & $3.90 \mathrm{a}$ & $6.27 \mathrm{a}$ & $15.17 \mathrm{a}$ & & Control & $3.02 \mathrm{a}$ & $2.98 \mathrm{a}$ & $2.69 \mathrm{a}$ & $3.26 \mathrm{a}$ & $8.93 \mathrm{a}$ & \\
\hline Treated & $3.15 \mathrm{a}$ & $2.16 \mathrm{~b}$ & $1.27 \mathrm{~b}$ & $2.40 \mathrm{~b}$ & $5.83 \mathrm{~b}$ & 62 & Treated & $2.30 \mathrm{a}$ & $1.04 \mathrm{a}$ & $0.90 \mathrm{~b}$ & $1.11 \mathrm{~b}$ & $3.05 \mathrm{~b}$ & 66 \\
\hline Base of trun & $\mathrm{nk}(10 \mathrm{~cm}$ & above gro & nd line) & & & & Base of trun & $k(10 \mathrm{~cm}$ & bove grou & ad line) & & & \\
\hline Control & $5.19 \mathrm{a}$ & $3.85 \mathrm{a}$ & $3.30 \mathrm{a}$ & $4.45 \mathrm{a}$ & $11.60 \mathrm{a}$ & & Control & $4.11 \mathrm{a}$ & $2.94 \mathrm{a}$ & $2.91 \mathrm{a}$ & $4.36 \mathrm{a}$ & $10.21 \mathrm{a}$ & \\
\hline Treated & $5.00 \mathrm{a}$ & $3.25 \mathrm{a}$ & $2.73 a$ & $2.25 \mathrm{~b}$ & $8.23 \mathrm{a}$ & 29 & Treated & $3.63 \mathrm{a}$ & $1.40 \mathrm{a}$ & $0.95 \mathrm{~b}$ & $1.16 \mathrm{~b}$ & $3.51 \mathrm{~b}$ & 66 \\
\hline Red oak & & & & & & & White pin & & & & & & \\
\hline Within cron & & & & & & & Within cron & & & & & & \\
\hline Control & $3.14 \mathrm{a}$ & $2.16 \mathrm{a}$ & $2.07 \mathrm{a}$ & $3.36 \mathrm{a}$ & $7.59 \mathrm{a}$ & & Control & $4.71 \mathrm{a}$ & $4.95 \mathrm{a}$ & $8.67 \mathrm{a}$ & $10.50 \mathrm{a}$ & $24.12 \mathrm{a}$ & \\
\hline Treated & $4.54 \mathrm{a}$ & $2.38 \mathrm{a}$ & $1.80 \mathrm{a}$ & $2.85 \mathrm{a}$ & $7.03 \mathrm{a}$ & 7 & Treated & $5.35 \mathrm{a}$ & $5.67 \mathrm{a}$ & $4.42 \mathrm{~b}$ & $5.29 \mathrm{~b}$ & $15.38 \mathrm{~b}$ & 36 \\
\hline Mid-trunk & (2-4 $\mathrm{mh}$ & & & & & & Mid-trunk & $2-4 m h i$ & & & & & \\
\hline Control & $4.32 \mathrm{a}$ & $2.82 \mathrm{a}$ & $2.37 \mathrm{a}$ & $3.38 \mathrm{a}$ & $8.57 \mathrm{a}$ & & Control & $9.77 \mathrm{a}$ & $9.05 \mathrm{a}$ & $10.39 \mathrm{a}$ & $10.77 \mathrm{a}$ & $30.21 \mathrm{a}$ & \\
\hline Treated & $4.17 \mathrm{a}$ & $2.23 \mathrm{a}$ & $1.79 \mathrm{a}$ & $2.60 \mathrm{a}$ & $6.62 \mathrm{a}$ & 23 & Treated & $8.48 \mathrm{a}$ & $8.79 \mathrm{a}$ & $8.42 \mathrm{~b}$ & $10.17 \mathrm{a}$ & $27.38 \mathrm{a}$ & 9 \\
\hline Base of trun & $\mathrm{nk}(10 \mathrm{~cm}$ & above gro & nd line) & & & & Base of trun & $k(10 \mathrm{~cm}$ & bove grou & nd line) & & & \\
\hline Control & $5.87 \mathrm{a}$ & $4.39 \mathrm{a}$ & $3.39 a$ & $4.91 \mathrm{a}$ & $12.69 \mathrm{a}$ & & Control & $9.17 \mathrm{a}$ & $11.32 \mathrm{a}$ & $9.84 \mathrm{a}$ & $12.08 \mathrm{a}$ & $33.24 \mathrm{a}$ & \\
\hline Treated & $5.97 \mathrm{a}$ & $3.19 \mathrm{~b}$ & $2.04 \mathrm{a}$ & $3.57 \mathrm{a}$ & $8.80 \mathrm{a}$ & 31 & Treated & $8.95 \mathrm{a}$ & $9.33 \mathrm{~b}$ & $8.22 \mathrm{a}$ & $11.46 \mathrm{a}$ & $29.01 \mathrm{a}$ & 13 \\
\hline Sweetgum & & & & & & & Yellow po & plar & & & & & \\
\hline Within crov & & & & & & & Within cron & & & & & & \\
\hline Control & $4.68 \mathrm{a}$ & $4.23 \mathrm{a}$ & $3.78 \mathrm{a}$ & $4.26 \mathrm{a}$ & $12.27 \mathrm{a}$ & & Control & $2.83 a$ & $2.64 \mathrm{a}$ & $2.98 \mathrm{a}$ & $3.65 \mathrm{a}$ & $9.27 \mathrm{a}$ & \\
\hline Treated & $4.18 \mathrm{a}$ & $1.99 \mathrm{~b}$ & $1.66 \mathrm{~b}$ & $1.54 \mathrm{~b}$ & $5.19 \mathrm{~b}$ & 58 & Treated & $2.74 \mathrm{a}$ & $1.92 \mathrm{a}$ & $2.57 \mathrm{a}$ & $3.83 \mathrm{a}$ & $8.32 \mathrm{a}$ & 10 \\
\hline Mid-trunk & $(2-4 m h$ & & & & & & Mid-trunk & $2-4 m h$ & & & & & \\
\hline Control & $4.50 \mathrm{a}$ & $4.42 \mathrm{a}$ & $3.51 \mathrm{a}$ & $3.58 \mathrm{a}$ & $11.51 \mathrm{a}$ & & Control & $3.28 \mathrm{a}$ & $2.55 \mathrm{a}$ & $2.71 \mathrm{a}$ & $3.99 \mathrm{a}$ & $9.25 \mathrm{a}$ & \\
\hline Treated & $3.54 \mathrm{a}$ & $2.46 \mathrm{~b}$ & $2.17 \mathrm{~b}$ & $2.07 \mathrm{a}$ & $6.70 \mathrm{~b}$ & 42 & Treated & $2.68 \mathrm{a}$ & $1.88 \mathrm{a}$ & $2.33 \mathrm{a}$ & $3.62 \mathrm{a}$ & $7.83 \mathrm{a}$ & 15 \\
\hline Base of trun & $\mathrm{nk}(10 \mathrm{~cm}$ & above gro & nd line) & & & & Base of trun & $k(10 \mathrm{~cm}$ & bove grou & ad line) & & & \\
\hline Control & $4.10 \mathrm{a}$ & $4.44 \mathrm{a}$ & $4.97 \mathrm{a}$ & $4.93 \mathrm{a}$ & $14.34 \mathrm{a}$ & & Control & $2.47 \mathrm{a}$ & $2.25 \mathrm{a}$ & $2.20 \mathrm{a}$ & $3.90 \mathrm{a}$ & $8.35 \mathrm{a}$ & \\
\hline Treated & $3.78 \mathrm{a}$ & $3.37 \mathrm{~b}$ & $3.45 \mathrm{~b}$ & $4.13 \mathrm{a}$ & $10.95 \mathrm{~b}$ & 24 & Treated & $2.25 \mathrm{a}$ & $2.16 \mathrm{a}$ & $2.63 \mathrm{a}$ & $4.16 \mathrm{a}$ & $8.95 \mathrm{a}$ & $(-7)$ \\
\hline
\end{tabular}

Pairs of values for each set of control and treated trees followed by the same lowercase letter are not significantly different at the $P \leq 0.05$ level.

treatment, resulting in a total reduction compared to untreated controls of $56 \%$ and $53 \%$, respectively. However, reduction of cambial growth in sweetgum occurred only in the first growing season after PBZ treatment, but this was sufficient to cause a $29 \%$ reduction at the base of trees during the two year period of the study (Table 6).

\section{DISCUSSION}

The principal focus of research with tree growth retardants has been on shoot length and growth in height. Reductions in shoot growth reported range from a low of $20 \%$ to a high of $90 \%$ among a broad spectrum of species including hardwoods, conifers, shrubs, and palms (Wheeler 1987; 
Table 6. Annual xylem ring increment $(\mathrm{mm})$ for the year prior to treatment and for 2 years after treatment with paclobutrazol in March 1997 using the soil drench method for cherrybark oak and sweetgum growing in Louisiana, with total reduction expressed as a percentage of untreated controls.

\begin{tabular}{|c|c|c|c|c|}
\hline \multirow[b]{3}{*}{ Treatment } & \multicolumn{2}{|r|}{ Year } & \multirow{3}{*}{$\begin{array}{l}\text { Total } \\
1997-98\end{array}$} & \multirow{3}{*}{$\begin{array}{l}\text { Percentage } \\
\text { reduction }\end{array}$} \\
\hline & Prior & After treatment & & \\
\hline & 1996 & 1997 & & \\
\hline
\end{tabular}

\section{Cherrybark oak}

Within crown (2-4 $m$ high)

Control $\quad 2.00$ a $\quad 2.22$ a $\quad 1.82$ a $\quad 4.04$ a

Treated $\quad 1.60 \mathrm{a} \quad 1.06 \mathrm{~b} \quad 0.82 \mathrm{~b} \quad 1.88 \mathrm{~b}$

At base of tree (10 cm above ground line)

Control $\quad 4.63$ a $\quad 5.86$ a $\quad 5.74$ a $\quad 11.60$ a

Treated $\quad 4.21 \mathrm{a} \quad 3.19 \mathrm{~b} \quad 1.89 \mathrm{~b} \quad 5.08 \mathrm{~b}$

\section{Sweetgum}

Within crown (2-4 $m$ high)

Control $\quad 1.75$ a $\quad 1.85$ a $\quad 0.99$ a $\quad 2.84$ a

Treated $\quad 1.29 \mathrm{a} \quad 1.52 \mathrm{~b} \quad 0.88 \mathrm{a} \quad 2.40 \mathrm{a}$

At base of tree (10 cm above ground line)

Control $\quad 2.91$ a $\quad 1.83$ a $\quad 1.76$ a $\quad 3.59$ a

Treated $\quad 2.01 \mathrm{a} \quad 1.21 \mathrm{~b} \quad 1.34 \mathrm{a} \quad 2.55 \mathrm{~b}$

29

Pairs of values followed by the same lowercase letter are not significantly different at the $P \leq 0.05$ level.

Ruter 1994; Burch et al. 1996; Hensley and Yogi 1996; Arron et al. 1997).

Of the nine species investigated in this study, only European black alder and cherrybark oak have not previously been reported to show reduction in shoot growth when treated with a gibberellin synthesis inhibitor like PBZ. Although shoot growth of European black alder was reduced by PBZ treatment, we could not quantify the amount because the terminal bud scale scars, which indicate the beginning and ending point of annual shoot growth, were not visible.

Our data demonstrate the wide variability among species in sensitivity to PBZ mentioned above. With the exception of sweetgum in Louisiana, only one dose rate was used for all nine species investigated. For example, shoot growth of black walnut was not affected by PBZ either in the main stem or in the lateral branches at the $9.6 \mathrm{~g}$ a.i. (0.34 oz.) dose rate. However, shoot growth in sweetgum at this dose rate was markedly reduced during the three growing seasons after treatment, whereas other species showed reductions in shoot growth in only some growing seasons (Tables 1, 2, and 3).

The differences in response among species found in this study are consistent with the dose rate charts provided by distributors of commercial formulations of PBZ that recommend six rates categories, A through $\mathrm{F}$ [1.25 to $4 \mathrm{~g}$ a.i. (0.04 to $0.14 \mathrm{oz})$ per inch $\mathrm{dbh}$ ] reflecting different sensitivities among species to PBZ. Black walnut, white ash, white pine, and yellow poplar are recognized as the least sensitive and are in category $\mathrm{F}$ for the highest dose rate. Sweetgum is quite sensitive to paclobutrazol and is in category B. Both red and white oak are intermediate and are in category D. Cherrybark oak, although not yet assigned to a category, probably should be in $\mathrm{D}$ based on the response found in this study. The majority of oaks are in category E, with a few in F. The dose rate applied in this study was that for category D or $\mathrm{E}$ depending on the diameter of the tree treated. Because of the small caliper $[4 \mathrm{~cm}(0.16 \mathrm{in})$.$] of the$ sweetgum trees used in Louisiana, the $4.8 \mathrm{~g}$ a.i. $(0.17 \mathrm{oz})$ dose rate also was that for category $\mathrm{E}$.

Although the preponderance of research has focused on shoot growth, a few observations have been recorded concerning growth in diameter. Schnurr et al. (1996) found that both PBZ and flurprimidol (another growth retardant with the same mode of action as PBZ) reduced height and stem caliper of Jack pine (Pinus banksiana) seedlings. Reduction in trunk diameter also was observed in apple (Malus spp.) (Estabrooks 1993), peach (Prunus spp.) (Liyembani and Taylor 1989), Colt cherry (Prunus avium 'P. pseudocerasus) (Asamoah and Atkinson 1985), and red maple (Acer rubrum) (Gilliam et al. 1988) with the suppression continuing for 2 to 3 years after treatment. The different responses in cambial growth we found among the species to the same dose of PBZ is consistent with the varied effects expected from a single dose rate on shoot growth.

Cambial activity at different vertical positions in a tree may respond to PBZ differently. For example, in white pine, reduction in xylem ring width occurred in the cross-section $10 \mathrm{~cm}$ (4 in.) above the ground line in the year of treatment. In the second year after treatment, reduction shifted to the cross-sections $2 \mathrm{~m}(6.6 \mathrm{ft})$ above the ground line, as well as within the canopy, and in the third year after treatment, xylem accumulation only in the uppermost cross-section was reduced.

This raises the question concerning the mode of action of PBZ on cambial growth. It was suggested by Elfving (1984) that the reduction in trunk growth in apple (Malus spp.) is due either to reduced shoot growth and associated leaf area or to direct effect of PBZ on cambial growth itself. Because gibberellins are not known to be produced in the cambium, but rather in leaf primordia and root tips, any effect on cell growth in the cambial region would rely on a disruption of the quantity of gibberellins translocated via the phloem or xylem (Leopold and Kriedemann 1975). The varied length of the translocation conduit between the gibberellin source and the cambial location in the trunk or tree crown, as well as the relative importance of leaf primordia or root tip produced gibberellins, could account for the differences reported. 
The climatic differences between Indiana and Louisiana seemed to have little influence on the efficacy of PBZ with respect to shoot growth (Tables 1, 2, and 3). However, there was a marked difference between the two experimental sites in cambial growth of sweetgum. At both locations, cambial growth was reduced in the year of treatment with PBZ, but in Louisiana the effect did not continue into the second growing season and the percent reduction was much less, both in the lower trunk and within the canopy in particular (Tables 5 and 6). This difference may be due to the application method, which was soil injection in Indiana and soil drench in Louisiana, rather than to climatic differences.

To provide a perspective of the potential impact of the reduction in shoot and cambial growth reported here on trees in the landscape, the total green aboveground weight of an untreated and PBZ treated white oak is estimated. The estimate is based on the average height $[750 \mathrm{~cm}(25 \mathrm{ft})]$ and trunk diameter [ $14 \mathrm{~cm}$ (5.6 in.)] of the control white oaks at the termination of this study. These average measurements were reduced by the 4-year reductions in height and diameter determined for white oak growing in Indiana (Tables 1 and 4). A nonlinear regression equation specific to white oak was used (Total green weight $=1.3426 \times$ $D^{2.2409} \times H^{0.4275}$ where $D$ is tree diameter and $H$ is height) (Myers and Polak 1976). Using this equation, the total weight of an average 16-year-old untreated white oak used in this study was found to be $109.3 \mathrm{~kg}$ (240 lb), whereas the total weight of a white oak treated with PBZ 4 years earlier was calculated to be $78.6 \mathrm{~kg}$ (173 lb), a $30.7 \mathrm{~kg}$ (67.6 lb) reduction in total weight.

Whether the reduction in cambial growth by PBZ was a function of a direct effect on meristematic activity and cell development through gibberellin synthesis inhibition or an indirect effect caused by reduced shoot growth with consequent reduced photosynthesis and carbohydrate partitioning was not determined and needs to be investigated. Additional studies also are needed to determine if there are any effects on cell structure, cell composition, or other micro aspects of xylem anatomy that could affect wood strength and susceptibility of trees to failure under wind or ice loading.

\section{LITERATURE CITED}

Arron, G.P., S. de Becker, H.A. Stubbs, and E.W. Szeto. 1997.

An evaluation of the efficacy of tree growth regulators paclobutrazol, flurprimidol, dikegulac, and uniconazole for utility line clearance. J. Arboric. 23:8-16.

Asamoah, T.E.O., and D. Atkinson. 1985. The effects of (2RS, 3RS)-1-(4-chlorophenyl)-4, 4-dimethyl-2-(1H, 2, 4 triazole-1-yl) pentan-3-ol (Paclobutrazol: PP333) and rootpruning on the growth, eater use and response to drought of Colt Cherry rootstocks. Plant Growth Regul. 3:37-45.
Burch, P.L., R.H. Wells, and W.N. Kline III. 1996. Red maple and silver maple evaluated 10 years after application of paclobutrazol tree growth regulator. J. Arboric. 22:61-66.

Chaney, W.R. 2003. Tree growth retardants: Arborists discovering new uses for an old tool. Tree Care Ind. 14(3):54-59.

Chaney, W.R., G.S. Premachandra, and H.A. Holt. 1996. Physiological basis for benefits of tree growth regulators, pp 8-18. In Proceedings, 8th Annual Conference of the Western Plant Growth Regulator Society, Sacramento, California, January 24-25, 1996.

Davis, T.D., and E.A. Curry. 1991. Chemical regulation of vegetative growth. Critical Rev. Plant Sci. 10:151-188.

Elfving, D.C. 1984. Control of apple tree growth and vigour with growth regulators. Compact Fruit Tree 17:142146.

Estabrooks, E.N. 1993. Paclobutrazol sprays reduce vegetative growth and increase fruit production in young McIntosh apple trees. Can. J. Plant Sci. 73:11271135.

Fletcher, R.A., A. Gilley, N. Sankhla, and T.D. Davis. 2000. Triazoles as plant growth regulators and stress protectants. Hortic. Rev. 24:55-138.

Gilliam, C.H., D.C. Fare, and J.T. Eason. 1988. Control of Acer rubrum growth with flurprimidol. J. Arboric. 14:99-101.

Grossmann, K. 1992. Plant growth retardants: Their mode of action and benefit for physiological research, pp 788-797 In Karssen, C.M., L.C. van Loon, and D. Vreugdenhil (Eds.). Progress in Plant Growth Regulation. Kluwer Academic Publishers, The Netherlands. 963 pp.

Hensley, D., and J. Yogi. 1996. Growth regulation of some tropical species. J. Arboric. 22:244-247.

Keever, G.J., W.J. Foster, and J.C. Stephenson. 1990. Paclobutrazol inhibits growth of woody landscape plants. J. Environ. Hortic. 8:41-47.

Kimball, S.L. 1990. The physiology of tree growth regulators. J. Arboric. 16:39-41.

Leopold, C.A., and P.E. Kriedemann. 1975. Plant Growth and Development (2nd ed.). McGraw-Hill, New York, NY. 545 pp.

Liyembani, S., And B.H. Taylor. 1989. Growth and development of young peach trees as influenced by foliar sprays of paclobutrazol or XE-1019. HortScience 24:65-68.

McPherson, E.G., and P.J. Peper. 1995. Infrastructure repair costs associated with street trees in 15 cities, pp 49-63. In Watson, G.W., and D. Neely (Eds.). Trees and Building Sites. International Society of Arboriculture, Champaign, IL.

Myers, C., and D. Polak. 1976. Full tree weight equations and tables for selected central hardwoods, pp 401-407. 
In Fralish, J.S., G.T. Weaver, and R.C. Schlesinger (Eds.). Proceedings of the Central Hardwood Forest Conference, Carbondale, IL, October 17-19, 1976.

Rademacher, W. 2000. Growth retardants: Effects on gibberellin biosynthesis and other metabolic pathways. Ann. Rev. Plant Physiol. Plant Mol. Biol. 51:501-531.

Redding, K.D., P.L. Burch, and K.C. Miller. 1994. Growth, biomass, and trim/chip time reduction following application of flurprimidol tree growth regulator. J. Arboric. 20:38-45.

Ruter, J.M. 1994. Growth and landscape establishment of Pyracantha and Juniperus after application of paclobutrazol. HortScience 29:1318-1320.

Schnurr, J.P., Z.-M. Cheng, and A.A. Boe. 1996. Effects of plant growth regulators on sturdiness of Jack pine seedlings. J. Environ. Hortic. 14:228-230.

Werblow, S. 1998. TGRs slow cycle buster trees. Vegetation Manage. J. 1(10):4-6.

Wheeler, N.C. 1987. Effect of paclobutrazol on Douglas fir and loblolly pine. J. Hortic. Sci. 62:101-106.

${ }^{1}$ Department of Computer Science

Southern University and A\&M College

P.O. Box 9221

Baton Rouge, LA 70813, U.S.

$2^{*}$ Department of Forestry and Natural Resources

715 West State Street

Purdue University

West Lafayette, IN 47907, U.S.

${ }^{3}$ Urban Forestry Program

Southern University and AEM College

P.O. Box 11288

Baton Rouge, LA 70813, U.S.

*Corresponding author.
Résumé. Trois expériences ont été menées afin de vérifier l'effet du paclobutrazol sur la croissance des pousses et du cambium de neuf espèces d'arbres localisées en Indiana et en Louisiane. Dans l'Expérience I, le chêne blanc (Quercus alba L.) et le chêne rouge (Quercus rubra L.) en Indiana ont été traités en avril 1995 avec du paclobutrazol au moyen de la méthode par trempage du sol. Dans l'Expérience II, le paclobutrazol a été appliqué en avril 1996 au moyen de la méthode par injection dans le sol sur huit espèces poussant en Indiana, soient le chêne blanc, le chêne rouge, le liquidambar styracifère (Liquidambar styraciflua L.), le noyer noir (Juglans nigra L.), l'aulne européen (Alnus glutinosa L.), le tulipier de Virginie (Liriodendront tulipifera L.), le frêne blanc (Fraxinus americana L.) et le pin blanc (Pinus strobus L.). Dans l'Expérience III, deux espèces en Louisiane-liquidambar et chêne rouge du Sud (Quercus falcata var. pagodaefolia L.) ont été traités par la méthode de trempage du sol en mars 1997. À la fin de la saison de croissance 1998, le taux de croissance de la pousse et l'épaisseur des anneaux de xylème ont été mesurés pour chacune des deux à quatre années de croissance qui se sont écoulées depuis le traitement avec les trois expériences. Les effets du paclobutrazol sur la croissance du cambium et des pousses annuelles, et ce à différentes positions sur le tronc et dans la cime, ont varié selon les espèces et la durée du traitement. Le paclobutrazol a diminué la croissance cambiale chez le chêne blanc, le chêne rouge, le chêne rouge du Sud, le liquidambar, l'aulne européen et le pin blanc de manière variable selon l'espèce, l'élévation dans l'arbre et le nombre d'Années après le traitement.

Zusammenfassung. Es wurden drei Experimente durchgeführt, um die Wirkung von Paclobutrazol auf das Trieb- und Kambiumwachstum von neun Baumarten in Indiana und Louisiana zu erforschen. In Experiment I wurden Quercus alba und Q. rubra in Indiana im April 1995 mit Paclobutrazol über den Boden durch Tränken behandelt. Im Experiment II wurde Paclobutrazol im April 
1996 durch Bodeninjektion an 8 Arten (Q. alba, Q. rubra, Liquidamber styraciflua, Juglans nigra, Alnus glutinosa, Liriodendron tulipifera, Fraxinus americana und Pinus strobus) in Indiana behandelt. Im Experiment III wurden im März 1997 in Louisiana 2 Baumarten (Liquidamber styraciflua und Q. falcata) durch eine Tränkung des Bodens behandelt. Am Ende der Wachstumsperiode 1998 wurden das jährliche Triebwachstum und der Jahresringzuwachs für jede der 2 bis 4 Wachstumsperioden nach der Behandlung gemessen. Die Auswirkungen von Paclobutrazol auf das sekundäre Dickenwachstum und die jährliche Trieblänge an verschiedenen Positionen entlang des Stammes und in der Krone variierte mit der Baumart und der Dauer der Behandlung. Paclobutrazol reduzierte das Dickenwachstum bei Q. alba, Q. rubra, Q. falcata, Liquidamber styraciflua, Alnus glutinosa und Pinus strobus in unterschiedlichem Ausmaß je nach Art, vertikaler Lage im Baum und dem Zeitraum nach der Behandlung.

Resumen. Se condujeron tres experimentos para investigar el efecto del paclobutrazol en el crecimiento cambial y de brotes de nueve especies de árboles localizados en Indiana y Louisiana. En el experimento I, el encino blanco (Quercus alba L.) y encino rojo (Q. rubra L.), en Indiana, fueron tratados en Abril de 1995 con paclobutrazol usando el método de zanjas en el suelo. En el experimento II, se aplicó paclobutrazol en Abril de 1996, usando el método de inyección al suelo, a ocho especies en Indiana: encino blanco, encino rojo, liquidámbar (Liquidambar styraciflua L.), nogal negro (Juglans nigra L), aile negro europeo (Alnus glutinosa L.), chopo amarillo (Liriodendron tulipifera L.), fresno blanco (Fraxinus americana L.) y pino blanco (Pinus strobus L.). En el experimento III, dos especies de liquidámbar y encino ( 2 . falcata var. pagodaefolia L.), en Louisiana, fueron tratadas con paclobutrazol por el método de zanjas en el suelo en Marzo de 1997. A finales de la estación de crecimiento de 1998 se midió el crecimiento de los brotes y el incremento de los anillos anuales del xilema para las cuatro estaciones de crecimiento después de los tratamientos en los tres experimentos. Los efectos del paclobutrazol en el crecimiento cambial y en los brotes, en diferentes localizaciones a lo largo del tronco y en la copa, variaron con las especies y la duración de los tratamientos. Paclobutrazol redujo el crecimiento cambial en encino blanco, encino rojo, liquidámbar, aile y pino blanco, en una cantidad variable de acuerdo a las especies, la localización vertical en el árbol y el año después del tratamiento. 